\title{
On the Transition to the Distant Learning as a Result of a Sudden External Shock: The Case of COVID 19 and HSE UrFU
}

\author{
Yeremenko V.I. ${ }^{1}$ Reprintseva N.E. ${ }^{2, *}$ Shishkin R.M. ${ }^{2}$
}

\author{
${ }^{1}$ Enterprise Center, Ural Federal University, Ekaterinburg, 620002, Russia \\ ${ }^{2}$ Higher School of Engineering, Ural Federal University, Ekaterinburg, 620002, Russia \\ *Corresponding author. Email: n.e.babinseva@gmail.com
}

\begin{abstract}
The impact of COVID 19 pandemic, even in the first sight, seems to be enormous. Our paper describes the third-level or tertiary reaction to the COVID 19 by the educational system of the Russian Federation. Following the government orders, as well as the university decrees, the transition process of the Higher School of Engineering Ural Federal University to the distant learning has met several obstacles. Description of those barriers as well as some recommendations on the reductions of the transition costs are the essential parts of our paper. We conclude that scenario analysis with several other tools might have been crucial for smoother transition to the online learning for all stakeholders.
\end{abstract}

Keywords: systems engineering, development of complex systems, engineering educational programs

\section{INTRODUCTION}

The work's relevance is due to the sharp transition to distance learning of students connected with the pandemic. Digital technology offers a range of opportunities that go beyond a temporary solution to a crisis. However, the COVID-19 crisis came when most systems are unprepared for the rise of digital learning opportunities. Currently, there is no model for a quick and large-scale transition to online education if full-time access to universities is closed.

The subject of research: difficulties encountered during the rapid transition from full-time education to distance learning, which influenced the effectiveness of the organization of the educational process / professional training of system engineers.

Tasks:

Consider the methods and forms of distance learning in the organization of the educational process.

Identify and analyze the difficulties encountered in the transition to distance learning.

Characterize the result and influence of this experience on the organization of the educational process.

Purpose of work: to study the experience of the rapid transition to distance learning and the organization of professional training at the university by using the case of the Higher School of Engineering at Ural Federal University. Develop proposals for a favorable transition to a distance learning system that quickly responds to various challenges.

Research methods: analysis of mass media, analysis of literature, analysis of the organization of the educational process, surveying students enrolled in the educational program 27.03.03 System analysis and management. When writing the article, the descriptive method was mainly used.

Results: a short introduction to the problems, principles, and methods of system engineering was made; the state of education in this area in Russia and abroad have been analyzed; the initiatives and the first experience of Tomsk Polytechnic University in the field of modernization of educational programs of the university in accordance with the principles of system engineering are considered.

Conclusions: The state of affairs on education in the field of system engineering in Russia can be assessed as catastrophic. Together with industry, Russian universities should take some decisive steps to introduce world experience in the field of teaching and applying system engineering. The implementation should take place in stages and continuously, based on the formation of standard programs and disciplines, taking into account the specifics of educational profiles. Elements of system engineering are expedient and necessary to be introduced into the training of bachelors. Elements of applied systems analysis can be built in the form of modules into existing undergraduate disciplines.

"Distance learning is characterized as a set of information technologies that ensure the delivery of the bulk of educational material to the learners, interactive interaction of learners and teachers in the learning process, providing students with the opportunity to independently work to master the material being studied, as well as the same opportunity, but already in the process of "contact" learning " [1]. 
The implementation of the distance education process is not unique, but during the pandemic, it exposed the most problematic areas in the implementation of vocational training. Because of the pandemic scale, a kind of testing of the educational system's readiness to respond to modern challenges took place. The need to form an educational model capable of quickly and favorably changing the form of student education in a pandemic influenced the analysis of the educational process's organization, a survey of students studying systems engineering.

The very concept of "flexible learning" is a model in which "online" and "offline" learning is combined with social interaction, where traditional pedagogical technologies are integrated with the achievements of electronic and distance learning [1]. In a flexible learning environment, the teacher must take on the role of a guide and encourage learners to be autonomous and self-reliant within the educational trajectory, taking into account the personalization of learning. [2].

In the current sanitary and epidemiological situation in the Russian Federation, the concept of "flexible learning" in educational institutions of the Ministry of Internal Affairs of Russia is not only relevant but also in demand, since it provides institutional conditions that are nonlinear, in which the implementation of educational programs carried out using e-learning and distance learning technologies. The effective use of flexible learning technology in education (e-learning) helps to adapt institutional systems, employees, and students to rapidly changing realities, flexibly managing the implementation of educational programs, and adapting to changes through the existing structures of the educational process.

In training future system engineers, special attention is paid to a project-based approach both in training and interaction with employers, and there are several good reasons for this:

- The direct involvement of employers gives the importance of the project movement.

- A situation is created to select motivated and capable students through a system of design competitions of increasing complexity.

- The most effective form of work organization at the stage of introducing new equipment and technologies is reproduced (in fact, projects of various kinds).

- The relevance of "competencies for competitiveness" increases for situations of new industries and the introduction of new technologies.

- The boundaries between purely scientific, purely engineering, and purely social projects are blurring. Thus, young engineers receive creative thinking, the ability to solve problems, critical thinking, and, of course, personal and interpersonal competencies - the ability to study independently, communicate effectively, work in a team, and projects. The involvement of students in the international project movement, the participation of project teams in various competitions - this is what is considered to be an essential component in the self-realization of an engineer all over the world.

- Project teams consist of students of different courses and different educational organizations, including representatives from enterprises, which makes it possible to identify a future specialist's inclinations.

\section{METHODOLOGY}

The response of HSE UrFU to the COVID 19 was not a direct one. To elaborate further, let us consider what happened from the department's point of view. First, there is a sudden external shock of coronavirus, which, fortunately, has not impacted the department directly. Second, as a part of the reaction by the federal and regional governments, the Ministry of Science and Higher Education issues several decrees and orders to protect the breach in the society's collective immunity. In particular, the order came that prescribed the universities to transfer all of its educational activities online and suspend all nonnecessary activities. The period of the order was only for one week at that time. Third, as a result of compliance with the recommendations and decrees, the university issues its decree describing the exact methods and procedures for such transition. Furthermore, lastly, as a part of the university, the department successfully managed to transfer educational activities for a week to the online. Of course, the action and the reaction have not ended there, as the quarantine and continuation of distant learning were prolonged until the end of the Spring semester of 2020. The issues that arose during that process of transition and our recommendations of avoiding or solving the said issues are to be discussed below. As one can see, the department can be seen as a tertiary reactant to the external shock of COVID 19. In our paper, we do not discuss the impact of secondary shock waves, such as the worldwide economic crisis and the epidemiological restrictions on society. Instead, we focus on one particular issue in education that helped us see how part of the educational system responded to sudden external shock and the reactions of the entire system to said shock.

\section{RESULTS OF THE STUDY}

Now, let us move onto more details of the issues the department encountered while transitioning to the distant learning format of education. A total of seven issues are analyzed, and the recommendations on how to avoid, predict, or solve such issues are discussed.

The first issue was the speed of the transition to distant learning. Although the university as a whole, and the department, in particular, had already transferred some of their courses to the online format, the mere challenge of transferring all of it, and in a short time was enormous. The communication between all of the learning process stakeholders had to be established, and the protocols of such communication had to be taught to all of the stakeholders. Both the faculty and the students had to be informed about the distant learning platforms, about the methods of conducting lectures and seminars, and about 
the evaluation processes of that one week (at that time) of distant learning.

The second issue was that after one week of the quarantine, the university had been put into a vacation for one week. After the exit from the vacation, the quarantine continued. Moreover, no one could predict when it will end, and when the classes will return to the normal offline mode. For instance, first, it was prolonged for another two weeks, then to another, then to the period on after the end of May holidays, and only after that to the end of the semester. As a result of such volatility and unpredictability, the entire educational process had to be continuously modified. The lecture content was updated continuously to allocate topics to be covered online and offline.

Third, the transition of the students to home-based learning also was seen as a problem. No one could be sure that all students had access to online tools and whether their internet connection was stable enough to accommodate the streaming of the lectures in real-time. Moreover, no one could predict whether all students' home conditions were suitable enough for learning itself.

Fourth, the faculty's transition to teaching from home was under the same scrutiny as the students' transition, but with more severe restrictions. One had to be sure that her internet connection was stable enough to upload the video stream and that the recording of the lectures would not be interrupted. Had the university been open to the instructors, it would not be an issue, but, alas, the quarantine was extended to the faculty.

The next problem that was foreseen but not dealt with was the students' lack of motivation. When transferred to the home environment, the students encountered many distractions to the online learning that were not present in the offline mode. The intragroup communications, the live contact with the faculty, and the limited amount of the classroom distractors were all missing in the distant learning. Added to the fact of the epidemiological danger of COVID 19 itself, it was understandable that the students became demotivated, and their participation in the learning activities declined.

Second to the last issue related to the fifth one was that control of the students' participation in the learning process had declined as well. While in the classroom, a competent lecturer always has the means to comprehend whether the student is listening or not, in the online mode, such comprehension is difficult. One can always use spot tests or another testing to check the students, but it takes time and extra effort to do that and is not possible in the large enough classes.

Last but not least was the problem of the change in the testing and exam protocols. In the classroom, anti-cheating measures are implemented more efficiently than in online mode. The online exams' proctoring is more expensive and can be circumvented more quickly than in the classroom setting. The need to rewrite the existing tests and exams to accommodate online anti-cheating protocols put more pressure on the faculty.

\section{ANALYSIS OF RESULTS}

In retrospection, the department's response to all of the issues mentioned above has been seen as more tactical than strategical. Although the goal of not decreasing the quality of education during the transition and the distant learning has always been in front of the department's minds, the result of the measures undertaken by HSE could have been more efficient. To rephrase Russian proverb: "If I were where I would fall, I would put a mat there." After analyzing the impact of the educational system's reaction to the COVID 19 pandemic to the department's educational process, we propose several recommendations to be considered in order to avoid or to decrease future external shocks.

First, the coronavirus news at the time of the system's reaction was not exactly new. The pandemic was declared at least a month earlier, and one could predict that the government will somehow react sooner or later. Our recommendation is to start conducting scenario analyses when dealing with something that might happen in the future. A couple of brainstorming sessions could pinpoint the weaknesses that could be resolved during quiet times before the possible impact of some external shock. Moreover, the planning for the worst possible scenarios could help the hedging for the impacts of more favorable scenarios.

Second, the exploration of alternative communication channels could help to resolve the students' home-learning processes. The occurrence of server crashes, especially during earlier stages of the transition, could be mitigated by using several alternatives to the lectures streaming. Zoom, Microsoft Teams, Google Meet, and several others could ensure that lectures were conducted on time. Using social media such as VK, Facebook, and messengers such as WhatsApp, Viber, Telegram, and others could help to make sure students always receive the actual information about all changes on time.

Third, to accommodate the faculty requests and ensure the quality of education received is not dropping, the regular online meeting with the course instructors should be conducted. That will help monitor the students' progress and monitor whether the instructors need additional help from the department before minor challenges disrupt the education process of particular instructors.

In order to increase the motivation of the students, several measures might be undertaken. Thus, the department asked the students to write an essay on the effect of the COVID 19 on their motivation, learning process, and financials. In the authors' opinion, such reflection of their issues helped students better embrace all the changes in their university experience. The analysis of the students' responses is currently work in progress and, hopefully, will be shared in future papers.

Our last recommendation is about the testing issues. We suggest that the educational programs' administration should educate and discuss with faculty the different testing methods. Switch from traditional tests to more research-oriented tasks such as research paper or online presentations might circumvent the possible cheating 
methods, as well as get a better comprehension of the student's progress and understanding of the topics covered through the course.

\section{CONCLUSION}

After analyzing the opinions of students of the Higher School of Engineering at UrFU about the current situation of a sudden transition to distance learning, we can highlight several fundamental conclusions: 1) the traditional form of education today is more promising, relevant, and of higher quality, in the opinion of the respondents, since it includes the essential part of the educational process - prompt communication with the teacher, the possibility of quick feedback, discussion of pressing issues, identification of gaps in their knowledge by communicating with the teacher in classroom lessons.

2) the main difficulties in the sudden transition from fulltime education to distance learning are the lack of face-toface communication with the teacher, the lack of dialogue between the two participants in the educational process, the lack of instant feedback since in order to answer the student's question of interest, the teacher spends much more time than in person. Also, remote feedback may depend not only on the two participants in the dialogue but on technical equipment, the absence of interruptions of the Internet, a server failure, or a power outage.

3 ) the advantages of switching to a distance form cannot be overlooked, the main of which was the flexibility of the educational process and a comfortable environment for students studying from home. Students note that a cozy home atmosphere makes them feel more relaxed, but at the same time does not distract from the perception of the educational material. Remembering the famous A. Maslow pyramid, where the very first level of satisfaction of needs is sleep and food, we can conclude that his pyramid works, because if a student is not full, he will not be able to perceive information. In a homely atmosphere where students can sit comfortably, take their favorite workplace, or pour themselves a cup of tea, it is natural that it is easier for them to perceive the material presented by the teacher. 4) mastering new programs, methods, technical knowledge of students is an absolute advantage of the distance learning format. Having switched to the distance form, students and teachers themselves are forced to look for the most convenient forms of conducting classes. For this, all free online platforms available in free access were studied, diagnosed, and tested, their tools and interface were considered in detail. Moreover, only then, having tried a lot of platforms and tools, the teacher and the student come to the choice of a perfect format of the training lesson. Thus, summing up all of the above, a more detailed examination of distance learning opens up a lot of additional opportunities for both the student and the teacher, on the one hand. On the other hand, the remote form of education is the relevance of today and, possibly, the future (a certain amount of hours in a particular discipline is possible in a distance format, for example, lectures). In our digital age, it is necessary to use all technological ideas and skills to keep up with the times. Not to replace the full-time teacher and full-time communication with him, but to correctly combine, combine all kinds of functions of technical progress and full-time education. And then there will be a modernization, improvement of the entire educational process and the goals set by the education system will be achieved.

\section{REFERENCES}

[1] A.A. Andreev, V.I. Soldatkin, Distance learning: essence, technology, organization. Publisher: MESI. M., 1991, P. 31

[2] I.N. Golitsina, Flexible learning in the traditional educational process, Higher education in Russia, 2017, No. 5, pp. 113-114.

[3] E. F. Crawley, Rethinking Engineering Education: The CDIO Approach, E. F. Crawley, J. Malmqvist, S. Östlund; Springer, 2014, P. 311.

[4] A. Kamp, Engineering Education in the Rapidly Changing World: Rethinking the Vision for Higher engineering Education, (2nd revised edition ed.) Delft: TU Delft, Faculty of Aerospace Engineering, 2016.

[5] A-Tuning-AHELO Conceptual Framework of Expected Desired, Learning Outcomes in Engineering, Electronic resource, OECD Publishing, Paris, 2011, P. 55. DOI: http://dx.doi.org/10.1787/5kghtchn8mbn-en.

[6] Aims of engineering education research - the role of the cdio initiative, Kristina Edström, Proceedings of the 12th International CDIO Conference, Turku University of Applied Sciences, Turku, Finland, June 12-16, 2016.

[7] O.I. Rebrin, What and how to teach modern engineers? /Rebrin O.I., Sholina I.I., Russian engineer, 2017, No. 2 (55), pp. 74-78.

[8] E. F. Crawley, The CDIO Syllabus v2. 0. An Updated Statement of Goals for Engineering Education, Electronic resource, E. F. Crawley, J. Malmqvist, W. A. Lucas, Proceedings of the 7th International CDIO Conference, Technical University of Denmark. - Copenhagen, 2011, P. 42.

[9] INCOSE 2012, Systems Engineering Handbook: A Guide for System Life Cycle Processes and Activities, version 3.2.2. San Diego, CA, USA: International Council on Systems Engineering (INCOSE), INCOSE-TP-2003002-03.2.

[10] A. Sage, W. Rouse, Handbook of Systems Engineering and Management, 2nd ed. Hoboken, NJ, USA: John Wiley and Sons, Inc., 2009. 
[11] EUR-ACE Framework Standards and Guidelines, Electronic resource, ENAEE, Edition 31st March, 2015, P. 26.

[12] A. Kamp, Impact of global forces and empowering situations on engineering education in 2030, A. Kamp, R. Klassen, Proceedings of the 12th International CDIO Conference, Turku: Turku University of Applied Sciences, 2016, pp. 1110-1120.

[13] O. Rebrin, Features of the modern educational environment for engineers, DAAAM International Scientific Book 2014, 2014, pp. 501-508.

[14] Engineering ontology. Engineering as a journey: a training manual, V. Nikitin, S. Pereslegin, A. Paribok, Yu. Chudnovsky, E. Pereslegin, N. Lukovnikova, D. Vasilkov, I. Tarikov, Yekaterinburg: LLC "Publishing House" Azhur", 2013, P. 230.

[15] Professionalism of a design engineer: analysis, evaluation and improvement: monograph, A. P. Isaev, A. M. Kozubsky, L. V. Plotnikov, G. G. Sukhanov, N. I. Fomin, V. O. Furin, Yekaterinburg: Publishing House Ural. University, 2015, P. 168 p.

[16] Evaluation of the training system for engineering personnel: materials of a comprehensive study of the needs of major regional employers, I. I. Sholina [et al.]; edited by L. N. Bannikova, Yekaterinburg: LLC "Publishing House" Azhur ", 2016, P. 272.

[17] The Greenfield era in education, SEDeC research, Moscow: Center for Educational Development of the Moscow School of Management Skolkovo, 2013, P. 52.

[18] O. I. Rebrin, New models of engineering education, University management: practice and analysis. - 2016, No. 102, pp. 61-71.

[19] A. Gibbs, Learning Outcomes, Degree Profiles, Tuning Project and Competences, Journal of the European Higher Education Area, 2012, Vol. 15, №5, pp. 71-87.

[20] O. I. Rebrin, Use of Learning Outcomes for Curriculum Design: Study guide, Vilnius: Ciklonas, 2016, P. 40.

[21] O. I. Rebrin, Collection of publications, Yekaterinburg: UrFU; LLC "Publishing House" Azhur", 2016, P. 168. 